\title{
Penthorum Chinense Pursh Protects Liver From Alcohol-induced Steatosis in Zebrafish by Mechanisms Including Inhibition of Oxidative Stress and Increase in Autophagy
}

\section{Xingtao Zhao}

Chengdu University of Traditional Chinese Medicine

\section{Mengting Zhou}

Chengdu University of Traditional Chinese Medicine

\section{Ying Deng}

Chengdu University of Traditional Chinese Medicine

\section{Chaocheng Guo}

Chengdu University of Traditional Chinese Medicine

\section{Li Liao}

chengdu university of traditional chinese medicine

\section{LinFeng He}

chengdu university of traditional chinese medicine

\section{Cheng Peng}

chengdu university of traditional chinese medicine

Yunxia Li ( $\square$ lyxtgyxcdutcm@163.com )

Chengdu University of Traditional Chinese Medicine

\section{Research}

Keywords: alcohol hepatosteatosis, Penthorum chinense Pursh, P2X7R, anti-oxidant, autophagy

Posted Date: December 30th, 2020

DOI: https://doi.org/10.21203/rs.3.rs-136206/v1

License: (c) (i) This work is licensed under a Creative Commons Attribution 4.0 International License. Read Full License 


\section{Abstract}

\section{Background}

As an ATP-gated ion channel, P2X7 receptor (P2X7R) affects lipid metabolism by activating the dangerous molecule ATP derived from liver cells caused by alcohol. Penthorum chinense Pursh (PCP), known as "shenxiancao", plays a significant role in treating liver disease among Miao people. We first investigated whether liver protection mechanism of PCP mediated by P2X7R.

\section{Methods}

First, treatment of zebrafish transgenic (fabp10: EGFP) larvae with different concentrations of PCP after $48 \mathrm{~h}$ at $3 \mathrm{dpf}$, then soaked in $350 \mathrm{mmol} / \mathrm{L}$ ethanol for $32 \mathrm{~h}$. Subsequently the ameliorative effect of PCP in zebrafish with alcoholic hepatosteatosis was studied. In addition, gene expression related to lipid metabolism, oxidative stress, and autophagy was detected from the mRNA level by RT-qPCR and related proteins were measured by Western blot. Then, larvae were stimulated with ATP alone to explore whether PCP was the target of P2X7R.

\section{Results}

PCP significantly improved liver function and lipid deposition in zebrafish with alcoholic hepatosteatosis, and regulated the expression of SREBP1, CHREBP and FAS by elevating LKB1 and AMPK, thereby inhibiting the synthesis of fatty acids. Also, SIRT1 was suppressed in the model group, while PCP upregulated the expression. Inecreased expression of PPARa, decreased PPARy, and CPT1 then promoted the oxidation of fatty acids. PCP dose-dependently decreased intracellular ROS production in zebrafish, then reduced MDA activity elevation and increased GSH, CAT and SOD levels. The specific mechanism may be realized by up-regulating the antioxidant pathway of Keap1/Nrf2 and down-regulating the autophagy pathway of PI3K/Akt/mTOR to regulate lipid metabolism. After ATP stimulation, P2X7R is further activated, which in turn regulated Keap1/Nrf2 and mTOR/PI3K/Akt mRNA expression, while PCP reversed these changes.

\section{Conclusions}

PCP may be a target of P2X7R involvement in the regulation of this mechanism through up-regulation of the antioxidant pathway of Keap1/Nrf2 and down-regulation of the autophagic pathway of mTOR/ $\mathrm{PI} 3 \mathrm{~K} /$ Akt to regulate lipid metabolism, suggesting that blocking P2X7R is an emerging strategy for the therapy of ALD.

\section{Highlight}

1. In vivo experiments showed that PCP improved liver function and reduced the steatosis of zebrafish induced by alcohol 
2. PCP modulated AMPK-Dependent steatosis in zebrafish by inhibiting the synthesis of fatty acids and promoting the oxidation of fatty acids

3. PCP mitigated alcohol hepatosteatosis by up-regulating the antioxidant pathway of Keap1/Nrf2 and down-regulating the autophagy pathway of mTOR/ PI3K/Akt signaling pathways

4. ATP-activated P2X7R were involved in regulating Nrf2-mediated oxidative stress and mTORmediated autophagy in zebrafish with alcohol hepatosteatosis

\section{Background}

Alcoholic liver disease (ALD) caused by heavy alcohol is currently highly prevalent worldwide and its liver damage has been well characterized[1], but there is no effective curative therapy in the clinical, so an indepth study of its mechanisms is necessary[2]. Lipid deposition in liver is the first stage of ALD, and then reactive oxygen species (ROS) is produced during the effects of ethanol metabolism, which causing the occurrence of oxidative-stress and autophagy[3]. However, the pathogenesis of ALD is not fully understood.

As an ATP-gated ion channel, $\mathrm{P} 2 \times 7$ receptor $(\mathrm{P} 2 \times 7 \mathrm{R})$ is present in an increasing number of different cell types, especially expressed in a variety of liver cells, including immune cells, HSC and liver cells[4]. P2 $\times$ $7 R$ accounts for a fairly vital role in health and disease, which can be activated by extracellular ATP to induce a variety of downstream events, including lipid metabolism, inflammatory molecule release, oxidative stress, cell proliferation and death, and autophagy[5]. Its receptor affects lipid metabolism[6-9] by activating the dangerous molecule ATP derived from liver cells caused by alcohol[10]. Recent studies have found that P2 $\times 7 R$ mediates the activation of NLRP3 inflamemome and lipid deposition in hepatocytes, P2 $\times 7 \mathrm{R}$ blocker alleviates alcohol-induced steatosis and intestinal flora changes via MEK1/2-ERK1/2 signaling[11]. Moreover, P2 $\times 7 R$ is a vital target in hepatitis-induced oxidative stressmediated autophagy, but the role of $\mathrm{P} 2 \times 7 \mathrm{R}$ in alcoholic liver remains to be further studied.

In order to further understand the pathogenesis of ALD, the development of rational targeted therapies is key to the clinical treatment or prevention of it. Penthorum chinense Push (PCP) (Ganhuangcao in Chinese), as a health food and folk medicine, is a plant from the family of Penthoraceae, which is traditionally used for hepatoprotection and hepatic diseases treatment, including alcoholic liver damage[12,13]. Recent pharmacological studies have shown PCP protects hepatocytes from ethanolinduced liver injury with anti-hepatic steatosis[14], antiaging[15], antiapoptotic[16], anti-inflammatory[17], and anti-oxidative stress[18]. While research on autophagy of PCP is minimal. We speculate that PCP may mediate alcohol-induced oxidative stress and autophagy in the liver. Therefore, further studies are needed.

Diseases such as neonatal cholestasis, cholangitis, polycystic liver disease, alcohol liver disease have been studied on zebrafish[19]. Thus, the present study was done to evaluate whether PCP could attenuate alcohol hepatosteatosis in zebrafish by regulating oxidative stress and autophagy related signaling pathway, which provides a new target for studying the mechanisms of alcohol hepatosteatosis. 


\section{Materials And Methods}

Chemicals and Reagents

The dried PCP, obtained from Chengdu HeHuaChi Chinese Herbal Medicine Market Co. (Sichuan, China) were extracted for three times by decoction, $1 \mathrm{~h}$ each time. After the solution is filtered, it is evaporated under reduced pressure to obtain a dry powder $17 \%(\mathrm{~g} / \mathrm{g})$ and stored at $-20^{\circ} \mathrm{C}$ for further use.

Oil red $O$ dyeing solution was collected from Shanghai Solarbio Bioscience and Technology Co., Ltd (Shanghai, China). DCF-DA was purchased from Yeasen Bio-technology Co., Ltd. (Shanghai, China). ATP disodium salt was purchased from MedChemExpress Co., Ltd. Rabbit AMPK (CS-5831), rabbit phosphoAMPK (CS-2535), rabbit PI3K(CS 4263S) and rabbit p-PI3K(CS 4228T) antibodies were from Celling Signaling Technology (MA, USA), rabbit ACC1 (AF6421), rabbit phosphor-ACC1 (AF3421), rabbit mTOR (AF6308), rabbit phosphor-mTOR (AF3309) and rabbit-GAPDH (AF0911) were purchased from Affinity Biosciences. Trizol reagents were purchased from Ambion Life Technologies (Carlsbad, CA, USA). All-Inone First-Strand cDNA SynthesisMix for qPCR (One-Step-gDNA Removal) was purchased from Beijing TransScript Biotechnology Co., Ltd. and Eva Green $2 \times$ RT-qPCR MasterMix-Low ROX was purchased from Applied Biological Materials Inc. (Richomnd, BC, Canada). PCR primer sequences were synthesized in TSINGKE Biological Technology (Chengdu, China). Other reagents used in the experiment were purchased from MultiSciences Biotech Co., Ltd.

Experimental animals and drug administration

Transgenic (fabp10: EGFP) zebrafish were obtained from China Zebrafish Resource Center (Wuhan, China), and raised at $28.5 \pm 1.0^{\circ} \mathrm{C}$ on a $14 \mathrm{~h}$ light/10 h dark cycle. Transgenic (fabp10: EGFP) zebrafish larvae at $3 \mathrm{dpf}$ were randomly assigned to 5 groups in a 6-well plate (30 larvae per well): larvae were maintained in filtered fish water as a control group while the model group was exposed to $350 \mathrm{mM}$ ethanol $(2 \% \mathrm{EtOH})$ for $32 \mathrm{~h}$ at $28.5^{\circ} \mathrm{C}[20]$. In PCP group, larvae were exposed to $48 \mathrm{~h}$ different doses PCP pre-treatment $(100 \mu \mathrm{g} / \mathrm{mL}, 50 \mu \mathrm{g} / \mathrm{mL}, 25 \mu \mathrm{g} / \mathrm{mL})$ followed by $350 \mathrm{mM}$ ethanol ( $2 \% \mathrm{EtOH})$ incubated in $28.5^{\circ} \mathrm{C}$ for $32 \mathrm{~h}$. ATP was given to further activate $\mathrm{P} 2 \times 7 \mathrm{R}$, and the experiment was divided into six groups, the control and model groups are treated in the same way as before and $1 \mathrm{mM}$ ATP for another 30 min at $28.5^{\circ} \mathrm{C}[21]$. In PCP group, larvae were exposed to $48 \mathrm{~h}$ different doses PCP pre-treatment $(100 \mu \mathrm{g} / \mathrm{mL}, 50 \mu \mathrm{g} / \mathrm{mL}, 25 \mu \mathrm{g} / \mathrm{mL})$ followed by $350 \mathrm{mM}$ ethanol ( $2 \%$ EtOH) for $32 \mathrm{~h}$, and $1 \mathrm{mM}$ ATP incubated in $28.5^{\circ} \mathrm{C}$ for another $30 \mathrm{~min}$. Afterwards, larvae were collected for detection.

Assessment of liver phenotype

After treatment, Zebrafish larvae were subjected to a series of pre-treatments including washed with fresh medium and subsequently anesthetized with tricaine, then fixed in $\mathrm{CMC}-\mathrm{Na}$, and adjusted to the lateral position. Then, zebrafish larvae were photographed under Leica M165Fic fluorescence microscope (Leica Microsystems, Germany). Finally, the fluorescence integral optical density of zebrafish livers were quantified using Image Pro Plus 6.0 software (Media Cybernetics, USA) was applied to quantify. 
After treatment, zebrafish larvae were collected and broken using an ultrasonic cell disruption system at $4{ }^{\circ} \mathrm{C}$ Triglyceride (TG) and total cholesterol (TC), alamine aminotransferase (ALT), aspartate aminotransferase (AST), Y-GT were obtained from Nanjing Jiancheng Bioengineering Institute (Nanjing, Jiangsu, China). After treatments, 30 larvae were cleaned by precooled PBS for three times, then homogenized and the supernatants were aspirated according to the instructions.

Whole-Mount Oil Red O Staining

Oil red 0 staining was used to determine hepatic lipid deposition. After treatment, zebrafish larvae were fixed overnight with 4\% PFA overnight. The rest of the procedure is routine[22]. Finally, the oil red 0 positive staining of zebrafish livers were photographed and related parameters was measured.

Assessment of ROS accumulation

2',7'-dichlorodihydrofluorescein diacetate (DCF-DA) was used as fluorescence probes to investigate intracellular production of ROS. Then operated according to instructions, the accumulation of ROS was measured.

Assessment of oxidative stress factors

Indicators of oxidative stress and oxidation resistance were measured. After treatment, zebrafish larvae were collected and broken using an ultrasonic cell disruption system at $4{ }^{\circ} \mathrm{C}$. Superoxide dismutase (SOD), malondialdehyde (MDA), reduced glutathione (GSH), catalase (CAT) assay kit was obtained from Elabsciense Bio-technology Co., Ltd. (Shanghai, China). After treatments, 30 larvae were cleaned by precooled PBS for three times, $270 \mu \mathrm{L}$ Slurry medium was added and homogenized, then the supernatants were aspirated according to the instructions.

Assessment the expression of related proteins via western blot analysis

At $4{ }^{\circ} \mathrm{C}$, RIPA lysis buffer containing PMSF and a protease inhibitor cocktail was used to extract zebrafish protein. Additional phosphatase inhibitors are required to extract of phosphorylated protein, then $50 \mu \mathrm{g}$ protein samples of p-AMPK, AMPK, PI3K, p-PI3K and p-ACC, ACC, p-mTOR, mTOR were determined using wester blotanalysis, the membranes were detected on a Tanon 5200 automatic chemiluminescence imaging analysis system (Shanghai, China) and quantified by Image-Pro Plus (version 6.0).

Assessment the expression of related genes via quantitative RT-qPCR

Trizol reagent was used to extract total RNA from zebrafish larvae and dissolved it in RNase-free water at $4{ }^{\circ} \mathrm{C}$. Ct values were obtained (reaction conditions: $95^{\circ} \mathrm{C} 10 \mathrm{~min}, 95^{\circ} \mathrm{C} 15 \mathrm{~s}, 60{ }^{\circ} \mathrm{C} 30 \mathrm{~s}(40 \mathrm{cycles})$ ) and the relative gene mRNA expression was determined on ABI7500 qPCR system and calculated using the $2^{-\triangle \Delta C t}$ method. The gene primer sequences used for RT-qPCR were listed in supplementary table 1. 
Statistical analyses

All values were expressed as means \pm SD. One-way analysis of variance (ANOVA) and Student's-test were used to assess the differences between the groups. The statistical analyses and graphs were generated by GraphPad Prism 6.0 (GraphPad, San Diego, CA, USA). Results were considered to be statistically significant when $\mathrm{p}<0.05$.

\section{Results}

Chemical characteristics of PCP extract

Previous studies showed that flavonoids were the main chemical constituents of PCP, which possess strong antioxidant activities[18]. The extract of PCP was analyzed by HPLC to determine its main chemical constituents, which was consistent with the previous studies that PCP extract contained a lot of flavonoids (Fig. 1). Six peaks were identified as gallic acid, rutin, quercetin, luteolin, apigenin and kaempferol. The contents of the six compounds were quantified using corresponding chemical standards. Specifically, the contents of gallic acid, rutin, quercetin, luteolin, apigenin and kaempferol in PCP were $1.2025,0.8244,0.4967,0.0924,0.026,0.0970 \mathrm{mg} / \mathrm{g}$, respectively.

PCP improved the liver function and attenuated hepatic accumulation

Liver fluorescence of Transgenic (fabp10: EGFP) zebrafish will be reduced after modeling (Fig. 2A), compared with the control group. We found that the liver fluorescence integral optical density (IOD) decreased significantly after modeling (Fig. 2B) and caused the remarkable increase of ALT, AST and $Y^{-}$ GT levels (Fig. 3A) $(P<0.01$ ), in addition, the rate of AST/ALT > 2 (Fig. 3A). PCP pretreatment strongly increased IOD in a dose-dependent manner, as well as decreased ALT, AST accumulation $(P<0.01)$, however only $100 \mu \mathrm{g} / \mathrm{mL}$ PCP pretreatment decreased $y-G T$ accumulation $(P<0.05)$, and significantly improved liver function $(P<0.01)$. Ethanol treatment caused a significant accumulation of $T G, T C$ concentrations, TG accumulation dominated (Fig. 3B). PCP pretreatment strongly reduced TG accumulation $(P<0.01)$ compared to those in the ethanol group in a dose-dependent manner, however only $100 \mu \mathrm{g} / \mathrm{mL}$ PCP pretreatment significantly reduced TC accumulation $(P<0.01)$. These results are consistent with whole-mount oil red $\mathrm{O}$ staining (Fig. 4), which demonstrated that PCP reduced liver lipid deposition.

PCP enhanced the level of oxidative stress-related factors

Drinking alcohol leads to the release of large amounts of intracellular ROS, as seen in Fig. 5A, a bright and strong fluorescent image was observed in the model group. PCP dose-dependently decreased intracellular ROS production in zebrafish (Fig. 5B). Compared with control group, the MDA levels of the ethanol-treated groups were significantly elevated (Fig. 6). Conversely, the levels of SOD, CAT and GSH markedly declined (Fig. 6). However, PCP pretreatment reduced the activity of MDA elevation and increased the activity of GSH and SOD in a dose-dependent manner, however only $100 \mu \mathrm{g} / \mathrm{mL}$ PCP 
pretreatment significantly improved the activity of CAT $(P<0.01)$. Thus, PCP alleviates alcohol hepatosteatosis by inhibiting oxidative stress.

PCP alleviated lipid deposition, oxidative stress and enhanced autophagy

mRNA expression of PCP in fatty acid synthesis and $\beta$-oxidation

To investigate the effect of PCP on fatty acid synthesis, as compared with control group, the upstream genes SIRT1, LKB1 and AMPK were decreased significantly in model group and reversed by PCP (Fig. 7A). RT-qPCR for the expression of four lipid metabolism-related genes, SREBP1, CHREBP, FAS, and ACC1. The results (Fig. 7C) showed that, mRNA SREBP1, CHREBP and FAS were increased significantly in model group and reversed by $100 \mu \mathrm{g} / \mathrm{ml}$ PCP in compared with control group $(P<0.001)$, and SREBP1 and FAS were decreased by $50 \mu \mathrm{g} / \mathrm{ml} \mathrm{PCP}(\mathrm{P}<0.001)$. However, there was no significant of ACC1 expression. Moreover, $\beta$-oxidation related genes including PPARa decreased, PPARY and CPT1 showed significantly increased in model group which can be reversed by $100 \mu \mathrm{g} / \mathrm{ml} \mathrm{PCP} \mathrm{(Fig.} \mathrm{7C)}(P<0.001)$ and $50 \mu \mathrm{g} / \mathrm{ml}$ PCP $(P<0.01)$, but no difference between the low doses compared with the model group $(p>0.05)$.

mRNA expression of PCP on anti-oxidant relevant signaling pathway

To further study the mechanism. Anti-oxidant effect of PCP was investigated with three related genes expression of keap1, Nrf2, HO-1 were examined by RT-qPCR. The results showed in Fig. 7E, indicated that, the levels of three increased genes in model group were significant in a dose-dependent decreased after $P C P$ administration $(P<0.001)$.

mRNA expression of PCP on autophagy relevant signaling pathway

Subsequently, RT-qPCR for detection of the autophagy effect of PCP was investigated with five mTOR signaling pathways related genes expression of mTOR, Atg13 and Beclin 1 and Upstream targets PI3K and Akt, and as shown in Fig. 6F, the expression of PI3K, Akt and mTOR increased, while Atg13 and Beclin 1 decreased significantly in model group $(P<0.001)$. Moreover, our data showed that the stimulation effect of alcohol hepatosteatosis in zebrafish was significantly reversed by high-concentrations of PCP $(\mathrm{P}<0.001)$, moreover PI3K, Akt, mTOR decreased in a concentration dependent manner after PCP administration.

PCP improved the liver function and attenuate hepatic accumulation

To further explore the underlying mechanism. Western blot experiments were conducted to detect the expression of related proteins, as shown in Fig. 8. In our study, there were no significant change in total proteins expression including AMPK, ACC and mTOR, mainly through phosphorylation, as the protein expression of $\mathrm{p}-\mathrm{AMPK} / \mathrm{AMPK}, \mathrm{p}-\mathrm{ACC} / \mathrm{ACC}$, and $\mathrm{p}-\mathrm{mTOR} / \mathrm{mTOR}$ were significantly decreased in model group $(P<0.001)$, which were significantly reversed by high-concentrations of $P C P(P<0.01)$, moreover, $p$ AMPK/AMPK and p-mTOR/mTOR increased in a dose-dependent manner after PCP administration and the results were consistent with the mRNA expression. Although, in the PCR results, we saw no significant 
change in mRNA expression of ACC, while the protein level PCP played its role mainly through phosphorylation of ACC. These data suggested that the possible mechanisms of PCP were primarily through phosphorylation of AMPK and mTOR targets.

ATP-activated P2 × 7R were involved in regulating oxidative stress and autophagy.

To further explore whether P2 $\times 7 \mathrm{R}$ mediated the possible mechanism of PCP inhibiting the oxidation and autophagy pathway, RT-qPCR for mRNA levels of P2 $\times 7 R$, AMPK, Keap1, Nrf2 oxidation-related, mTOR, $\mathrm{PI} 3 \mathrm{~K}$, and Akt autophagy-related genes. Compared with the ethanol treatment group, the mRNA expression of P2 $\times 7 R$ in the ethanol plus ATP group was significantly increased (Fig. 9A-1), then PCP significantly decreased its expression compared with both control and ethanol group in a dose-dependent manner. In the ethanol plus ATP group, activated P2 $\times 7$ R further increased the expression of Keap1 (Fig. 9A-3) related to oxidative stress, and significantly reduced the expression of AMPK and Nrf2 (Fig. 9A$2,4)$, moreover, compared with the ethanol plus ATP treatment group, mTOR, PI3K, Akt decreased, however PCP treatment significantly reversed these in a dose-dependent. More interestingly, mRNA expression of PI3K and Akt significantly decreased $(P<0.001)$ compared with ethanol plus ATP group (Fig. 9B).

\section{Discussion}

Serum levels of ALT and AST are vital and sensitive biochemical signals of liver function, indicating early stages of ALD, whose abnormal elevation can cause liver cell damage and necrosis[23]. In addition, $\gamma^{-}$ GGT has been used as a marker of hepatic impairment due to alcohol consumption[24]. Consistent with our results, compared with the model group, different doses of PCP effectively reduced ALT, AST and Y-GT levels and improved liver function. Moreover, during the pathological development of ALD, the most common phenomenon is an imbalance between lipid synthesis and fatty acid oxidation. Hepatic steatosis as a reversible process that can exacerbate disease progression[25]. AMPK signaling plays a meaningful role in lipid homeostasis regulation, as a highly conserved sensor for low intracellular ATP levels, which is produced in large amounts in alcoholic fatty liver. P2 $\times 7 \mathrm{R}$ is a vital regulatory point for transmitting extracellular ATP signals that allow AMPK to be activated. It has been reported that dihydroquercetin mediates P $2 \times 7 \mathrm{R}$ amelioration of alcoholic hepatic steatosis[8]. In addition, AMPK phosphorylation regulates downstream ACC, where ACC is a key enzyme in fatty acid synthesis, catalyzing acetyl coenzyme A carboxylation to malonyl coenzyme $A$, and then regulating PPAR-a and CPT1 pathway promoting free fatty acid oxidation; PPARY showed the opposite pharmacological effect. moreover, SREBP-1c is a fat synthesis protein (e.g., FAS), which is the primary regulator of triglyceride conversion and decreased lipogenesis, TG accumulation by upregulating AMPK signaling by SREBP-1C and by downregulating FAS.

Oxidative stress is a key point in the process of ALD[26]. Alcohol consumption leads to high ROS production, suppresses antioxidant defense systems, and leads to oxidative stress in the liver[27]. Lipid peroxidation products (e.g., MDA) indirectly reflects the extent of free radical damage to the liver, and it is also an indicator of hepatocyte recovery after PCP administration. In contrast, the antioxidant defense 
systems GSH, CAT and SOD, which directly reflect the antioxidant capacity of the liver, remove of lipid peroxides and protect hepatocytes from ROS damage, while PCP treatment significantly reduced ROS. MDA level was decreased and increased SOD, GSH and CAT level in our study. Moreover, P2 $\times 7 \mathrm{R}$ activation induces ROS production and antioxidative defense system imbalances[28], which is key factors in oxidative stress-driven cell fate[29], and the downstream AMPK prevents oxidative stress primarily by regulating glucose and lipid metabolism[30], which activates Nrf2. Nrf2 is the key to cellular resistance to ROS, and studies suggest that targeting Keap1/Nrf2 can effectively reduce oxidative stress[31], and related studies have also found that AMPK mediates oxidative stress by increasing intracellular levels of its co-substrate, $\mathrm{NAD}^{+}$, and that hyperphosphorylation of AMPK inhibits Nrf2 activation and nuclear translocation by modifying cysteine residues in Keap1, which in turn inhibits oxidative stress by inducing the expression of various antioxidant genes, such as HO-1[32]. Consistent with our experimental results, we observed Keap1 was increased and AMPK, Nrf2 was decreased in the model group at the gene level, while Nrf2 was increased after administration of PCP indicating the inhibition of oxidative stress, and P2 $\times 7 R$ was activated by ATP and was involved in Keap1/Nrf2mediated oxidative stress, which suggests that $P 2 \times 7 R$ may be involved in lipid metabolism via Keap1/Nrf2-mediated oxidative stress.

Recently, autophagy has become a protective mechanism against ALD, although there are currently divergent mechanisms in acute and chronic alcohol-induced alcoholic hepatitis[33-35]. Some studies found that P2 $\times 7 R$ played an important role in ALD, signals from P2 $\times 7 R$ and adipoR1 regulate the PI3KAkt and/or AMPK-Foxo3A pathway to restore the mitochondrial autophagy (mitosis) inhibited by ethanol intoxication[36]. Moreover, as the upstream target of PI3K/Akt, mTOR plays a vital role in autophagy, which mediates autophagy may be a potential mechanism for treating liver injury[37]. In our results, at the genetic level we observed that the administration of mTOR was able to reverse the expression of mTOR compared to the model group and further verified from the protein level that it acted mainly through phosphorylation of the expression. In addition, this paper also observed that the classical autophagy signaling pathway mTOR/PI3K/Akt was altered after activation of the P $2 \times 7 \mathrm{R}$ by administration of ATP, and PI3K/Akt changed significantly.

Moreover, there is some crosstalk between oxidative stress and autophagy. They can work together to participate in lipid metabolism, $\mathrm{P} 2 \times 7 \mathrm{R}$ plays a key role in this process, suggesting that P2 $\times 7 \mathrm{R}$ is a vital mediator of alcoholic hepatitis, mediates the development of alcoholic hepatitis, and is a new target for alcoholic hepatitis treatment. PCP was first used in an ALD zebrafish model to investigate the beneficial effects of alcohol via P2 $\times 7 \mathrm{R}$ in mediating lipid metabolism, oxidative stress and autophagy (Fig. 10). The mechanism of PCP in alcoholic liver was revealed more comprehensively, which provided a new idea for the treatment of alcoholic liver.

\section{Conclusion}

In summary, P2 $\times 7$ discovery provides a new target for potential pathways of ALD. PCP may be a target of $\mathrm{P} 2 \times 7 \mathrm{R}$ involvement in the regulation of this mechanism through up-regulation of the antioxidant 
pathway of Keap1/Nrf2 and down-regulation of the autophagic pathway of mTOR/ PI3K/Akt to regulate lipid metabolism.

\section{Abbreviations}

ACC, acetyl coA carboxylase; ALD, alcohol liver disease; ALT, alanine transaminase; AMPK, adenosine monophosphate protein kinase protein; ASH, alcoholic steatohepatitis; AST, aspartate transaminase; Atg13, autophagy related gene 13; CAT, Catalase; ChREBP, carbohydrate response element-binding protein; DCF-DA, 2',7'-dichlorodihydrofluorescein diacetate; dpf, days post fertilization; FAS, fatty acid synthetase; GSH, glutathione; $\gamma$-GT, y-glutamyltransferase; IOD, integral optical density; Keap1, kelch like ECH associated protein1; LKB1, liver kinase b1; MDA, malondialdehyde; mTOR, mechanistic target of rapamycin; NQ01, NAD $(P) H$, quinine oxidoreductase 1; Nrf2, nuclear Factor E2 related factor 2; PCP, Penthorum chinense Pursh; P2X7R, P2x7 receptor; PI3K, the class III phosphatidylinositol-3-kinase; PPAR, peroxisome proliferator activated receptor; ROS, reactive oxygen species; RT-qPCR, quantitative Real-Time PCR; SIRT1, Sirtuin 1; SOD, superoxide dismutase; SREBP1, sterol-regulatory element binding protein 1; TC, total cholesterol; TG, total triglyceride.

\section{Declarations}

\section{Ethics approval and consent to participate}

The experiments involving larval zebrafish were performed under the approval of the Institutional Animal Care and Use Committee of Chengdu University of Traditional Chinese Medicine (No. SYXK (CHUAN) 2014-128).

\section{Consent for publication}

All the authors read the final manuscript and approved for publication.

\section{Availability of data and materials}

The datasets used in this study are available from the corresponding author upon reasonable request.

\section{Competing interests}

The authors declare that there are no conflicts of interests

\section{Funding}


National Science Foundation of China (Grant No. 81373943, 81573583, U19A2010)

Sichuan Provincial Science Technology Department of Youth Science and technology innovation research team program (Grant No. 2017TD0001)

Xinglin Scholar Research Premotion Project of Chengdu University of TCM (Grant No. CXTD2018019)

\section{Authors' contributions}

X.Z. (Xingtao Zhao), Y.L. (Yunxia Li) and C.P. (Cheng Peng) conceived and designed the experiments. X.Z., M.Z., Y.D., C.G., L.L., M.L., and L.H., conducted the biological experiments and analyzed the data. X.Z. and Y.L. wrote the manuscript. C.P. provided expert technical support and critical revision of the manuscript for important intellectual content and language editing.

\section{Acknowledgements}

This work was financially supported by the research was supported by National Science Foundation of China (Grant No. 81373943, 81573583, U19A2010), Sichuan Provincial Science Technology Department of Youth Science and technology innovation research team program (Grant No. 2017TD0001), and Xinglin Scholar Research Premotion Project of Chengdu University of TCM (Grant No. CXTD2018019)

\section{References}

1. S.K. Asrani, H. Devarbhavi, J. Eaton, P.S. Kamath, Burden of liver diseases in the world, J Hepatol 70(1) (2019) 151-171.

2. M. You, G.E. Arteel, Effect of ethanol on lipid metabolism, Journal of hepatology 70(2) (2019) 237248.

3. G. Filomeni, D. De Zio, F. Cecconi, Oxidative stress and autophagy: the clash between damage and metabolic needs, Cell Death Differ 22(3) (2015) 377-88.

4. X. Li, Y. Zhang, Liver kinase B1/AMP-activated protein kinase-mediated regulation by gentiopicroside ameliorates $\mathrm{P} 2 \mathrm{X} 7$ receptor-dependent alcoholic hepatosteatosis, British journal of pharmacology 175(9) (2018) 1451-1470.

5. R. Sluyter, The P2X7 Receptor, Advances in experimental medicine and biology 1051 (2017) 17-53.

6. J. Suurvali, P. Boudinot, J. Kanellopoulos, S. Ruutel Boudinot, P2X4: A fast and sensitive purinergic receptor, Biomed J 40(5) (2017) 245-256.

7. S. Jiang, Y. Zhang, J.H. Zheng, X. Li, Y.L. Yao, Y.L. Wu, S.Z. Song, P. Sun, J.X. Nan, L.H. Lian, Potentiation of hepatic stellate cell activation by extracellular ATP is dependent on P2X7R-mediated NLRP3 inflammasome activation, Pharmacol Res 117 (2017) 82-93. 
8. Y. Zhang, Q. Jin, Amelioration of Alcoholic Liver Steatosis by Dihydroquercetin through the Modulation of AMPK-Dependent Lipogenesis Mediated by P2X7R-NLRP3-Inflammasome Activation, Journal of agricultural and food chemistry 66(19) (2018) 4862-4871.

9. X. Li, Q. Jin, Y. Zhang, Y.L. Wu, C.M. Jin, B.W. Cui, Y. Li, M.J. Jin, Y. Shang, M. Jiang, H.X. Yang, M. Wu, J. Liu, L.H. Lian, J.X. Nan, Inhibition of P2X7R-NLRP3 Inflammasome Activation by Pleurotus citrinopileatus: A Possible Protective Role in Alcoholic Hepatosteatosis, J Agric Food Chem 66(50) (2018) 13183-13190.

10. J. Petrasek, A. Iracheta-Vellve, Metabolic danger signals, uric acid and ATP, mediate inflammatory cross-talk between hepatocytes and immune cells in alcoholic liver disease, Journal of leukocyte biology 98(2) (2015) 249-56.

11. Q.Q. Su, Y.Y. Tian, Purinergic P2X7 receptor blockade mitigates alcohol-induced steatohepatitis and intestinal injury by regulating MEK1/2-ERK1/2 signaling and egr-1 activity, International immunopharmacology 66 (2019) 52-61.

12. A. Wang, M. Li, A review of Penthorum chinense Pursh for hepatoprotection: Traditional use, phytochemistry, pharmacology, toxicology and clinical trials, Journal of ethnopharmacology 251 (2020) 112569.

13. X. Zhao, L. Li, M. Zhou, M. Liu, Y. Deng, L. He, C. Guo, Y. Li, An Overview of the Mechanism of Penthorum chinense Pursh on Alcoholic Fatty Liver, Evidence-based complementary and alternative medicine : eCAM 2020 (2020) 4875764.

14. W.W. Guo, X. Wang, X.Q. Chen, Y.Y. Ba, N. Zhang, R.R. Xu, W.W. Zhao, X. Wu, Flavonones from Penthorum chinense Ameliorate Hepatic Steatosis by Activating the SIRT1/AMPK Pathway in HepG2 Cells, Int J Mol Sci 19(9) (2018).

15. D. Jeong, J. Lee, S.H. Park, Y.A. Kim, B.J. Park, J. Oh, G.H. Sung, A. Aravinthan, J.H. Kim, H. Kang, J.Y. Cho, Antiphotoaging and Antimelanogenic Effects of Penthorum chinense Pursh Ethanol Extract due to Antioxidant- and Autophagy-Inducing Properties, Oxid Med Cell Longev 2019 (2019) 9679731.

16. X. Sun, A. Wu, The active components derived from Penthorum chinensePursh protect against oxidative-stress-induced vascular injury via autophagy induction, Free radical biology \& medicine 146 (2020) 160-180.

17. L.M. Lin, L.J. Zhao, J. Deng, S.H. Xiong, J. Tang, Y.M. Li, B.H. Xia, D.F. Liao, Enzymatic Extraction, Purification, and Characterization of Polysaccharides from Penthorum chinense Pursh: Natural Antioxidant and Anti-Inflammatory, Biomed Res Int 2018 (2018) 3486864.

18. Y. Hu, S. Wang, Antioxidant and hepatoprotective effect of Penthorum chinense Pursh extract against t-BHP-induced liver damage in L02 cells, Molecules (Basel, Switzerland) 20(4) (2015) 6443-53.

19. D.H. Pham, C. Zhang, C. Yin, Using zebrafish to model liver diseases-Where do we stand?, Curr Pathobiol Rep 5(2) (2017) 207-221.

20. D.L. Howarth, M. Passeri, Drinks like a fish: using zebrafish to understand alcoholic liver disease, Alcoholism, clinical and experimental research 35(5) (2011) 826-9. 
21. F.F. Cruz, C.E. Leite, Assessment of mercury chloride-induced toxicity and the relevance of P2X7 receptor activation in zebrafish larvae, Comparative biochemistry and physiology. Toxicology \& pharmacology : CBP 158(3) (2013) 159-64.

22. L. Yu, L. Gong, Radix Polygoni Multiflori and Its Main Component Emodin Attenuate Non-Alcoholic Fatty Liver Disease in Zebrafish by Regulation of AMPK Signaling Pathway, Drug design, development and therapy 14 (2020) 1493-1506.

23. Y.W. Cao, Y. Jiang, Protective effects of Penthorum chinense Pursh against chronic ethanol-induced liver injury in mice, Journal of ethnopharmacology 161 (2015) 92-8.

24. J.B. Whitfield, Gamma glutamyl transferase, Critical reviews in clinical laboratory sciences $38(4)$ (2001) 263-355.

25. E.S. Orman, G. Odena, Alcoholic liver disease: pathogenesis, management, and novel targets for therapy, Journal of gastroenterology and hepatology 28 Suppl 1(0 1) (2013) 77-84.

26. S. Seeland, H. Kettiger, ATP-induced cellular stress and mitochondrial toxicity in cells expressing purinergic P2X7 receptor, Pharmacology research \& perspectives 3(2) (2015) e00123.

27. H. Zhu, Z. Jia, Oxidative stress and redox signaling mechanisms of alcoholic liver disease: updated experimental and clinical evidence, Journal of digestive diseases 13(3) (2012) 133-42.

28. B. Wang, R. Sluyter, $P 2 X 7$ receptor activation induces reactive oxygen species formation in erythroid cells, Purinergic signalling 9(1) (2013) 101-12.

29. S. Chatterjee, S. Das, P2X7 receptor as a key player in oxidative stress-driven cell fate in nonalcoholic steatohepatitis, Oxidative medicine and cellular longevity 2015 (2015) 172493.

30. K. Fang, F. Wu, Diosgenin ameliorates palmitic acid-induced lipid accumulation via AMPK/ACC/CPT$1 \mathrm{~A}$ and SREBP-1c/FAS signaling pathways in LO2 cells, BMC complementary and alternative medicine 19(1) (2019) 255.

31. H.K. Tan, E. Yates, Oxidative stress in alcohol-related liver disease, World journal of hepatology 12(7) (2020) 332-349.

32. R. Li, Y. Liu, Bailcalin Protects against Diabetic Cardiomyopathy through Keap1/Nrf2/AMPKMediated Antioxidative and Lipid-Lowering Effects, Oxidative medicine and cellular longevity 2019 (2019) 3206542.

33. L. Yang, D. Wu, Cytochrome P4502E1, oxidative stress, JNK, and autophagy in acute alcohol-induced fatty liver, Free radical biology \& medicine 53(5) (2012) 1170-80.

34. W.X. Ding, M. Li, Autophagy reduces acute ethanol-induced hepatotoxicity and steatosis in mice, Gastroenterology 139(5) (2010) 1740-52.

35. W.X. Ding, M. Li, Selective taste of ethanol-induced autophagy for mitochondria and lipid droplets, Autophagy 7(2) (2011) 248-9.

36. H. Gao, Y. Lv, Wolfberry-Derived Zeaxanthin Dipalmitate Attenuates Ethanol-Induced Hepatic Damage, Molecular nutrition \& food research 63(11) (2019) e1801339. 
37. H. Wang, Y. Liu, The Upstream Pathway of mTOR-Mediated Autophagy in Liver Diseases, Cells 8(12) (2019).

\section{Figures}
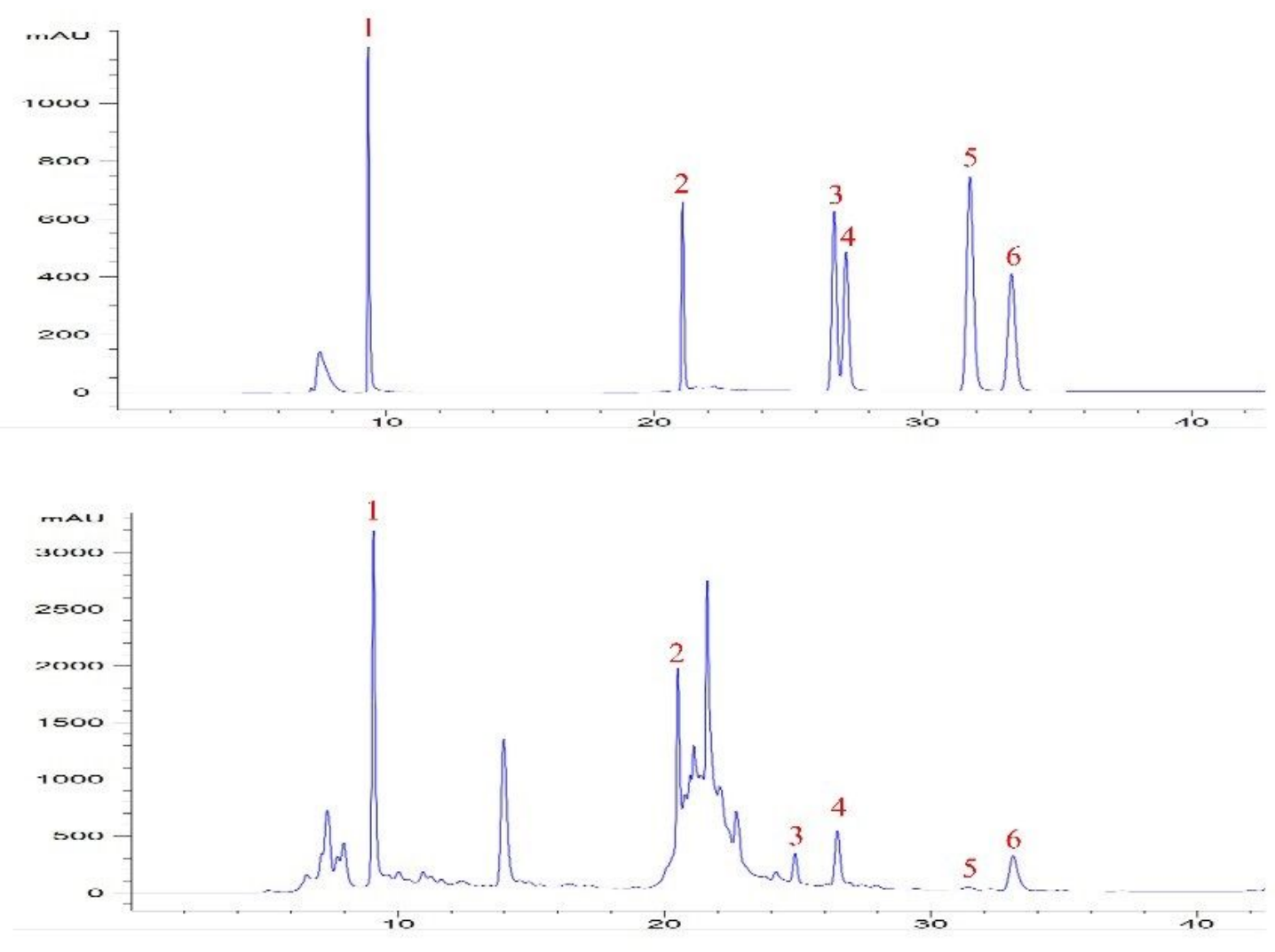

\section{Figure 1}

Representative HPLC-UV chromatograms of mixed standards and P. chinense extract. Gallic acid (1), lutin (2), quercetin (3), luteolin (4), apigenin (5) and kaemferol (6) 
A
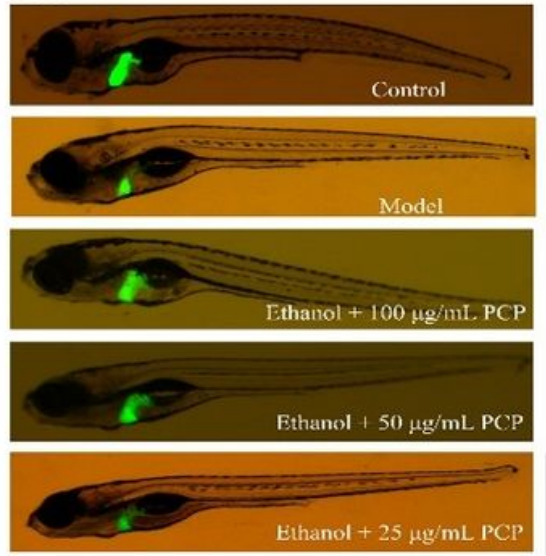
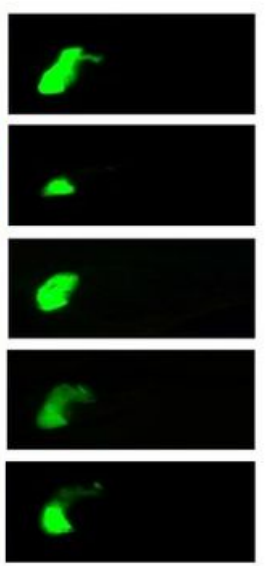

B

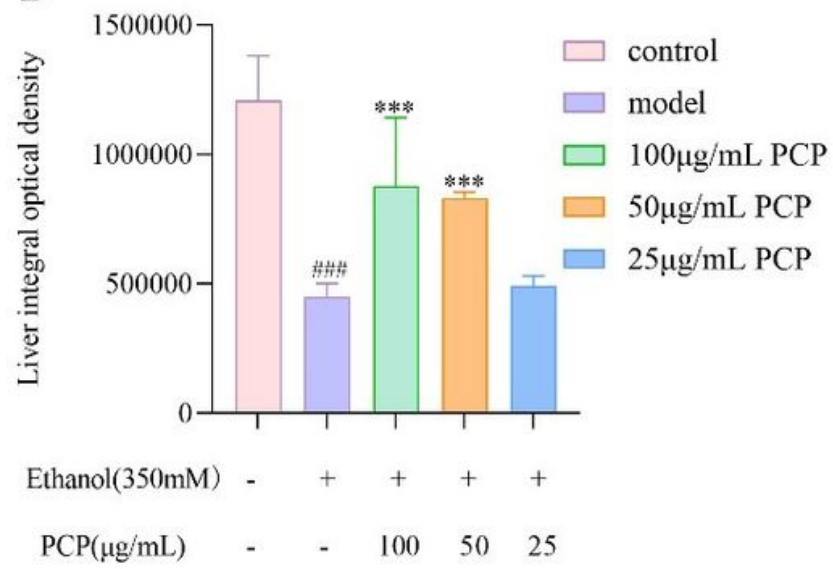

Figure 2

Different doses of PCP effect on serum biochemical indicators of liver function. (A) The fluorescence intensity of the liver part; (B) The percent of integrated optical density (IOD) of the area (statistical analysis by Image Pro Plus 6.0 software). 

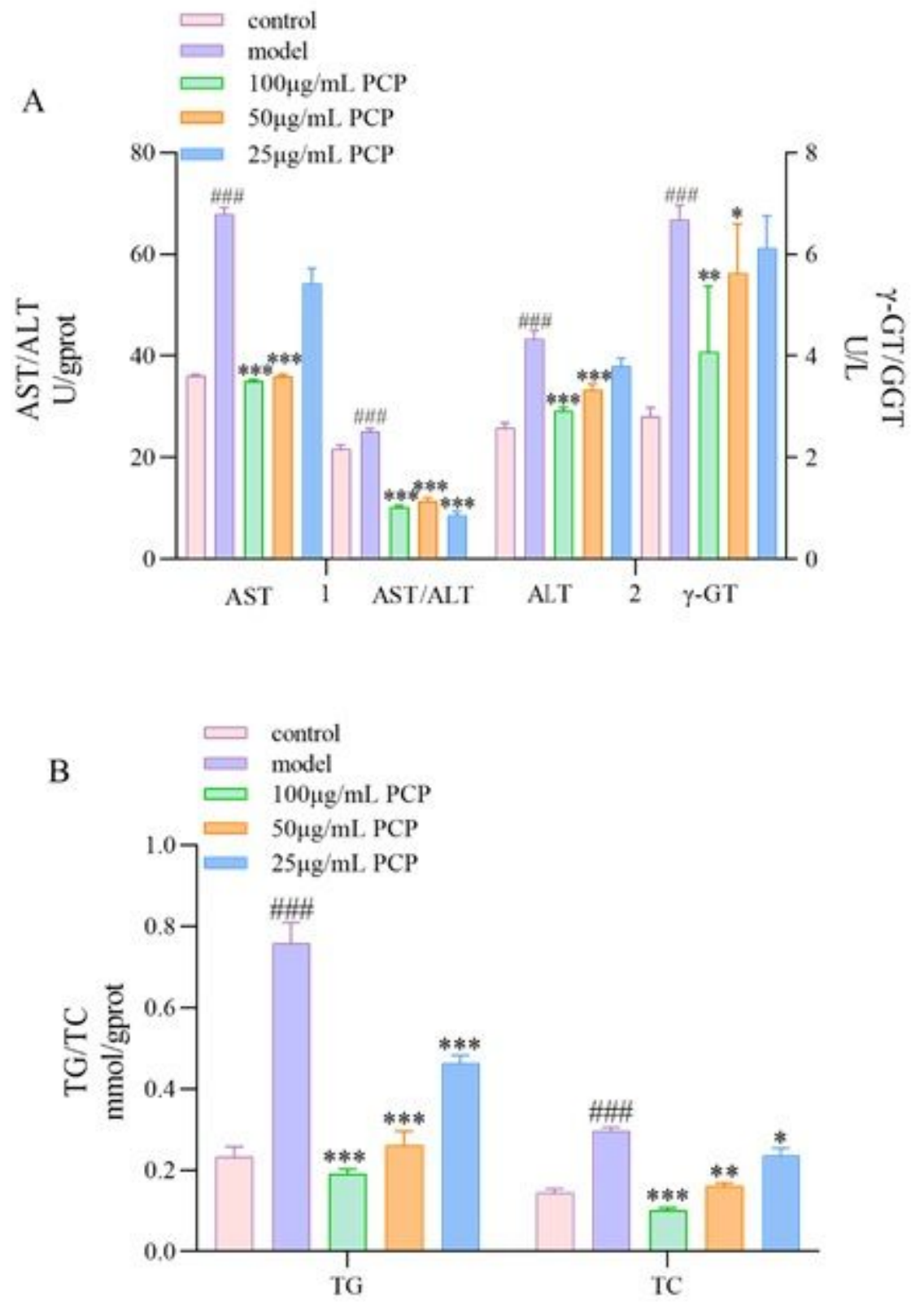

\section{Figure 3}

Different doses of PCP effect on serum biochemical indicators of liver function. The following six liver function markers in the tissue were assayed: (A) ALT; AST; ALT/AST; $\mathrm{Y}-\mathrm{GT}$; (B) TG; TC. Data expressed as

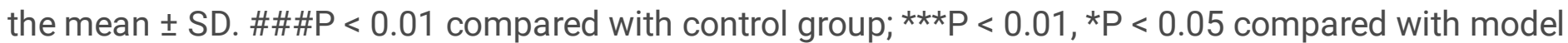
group 

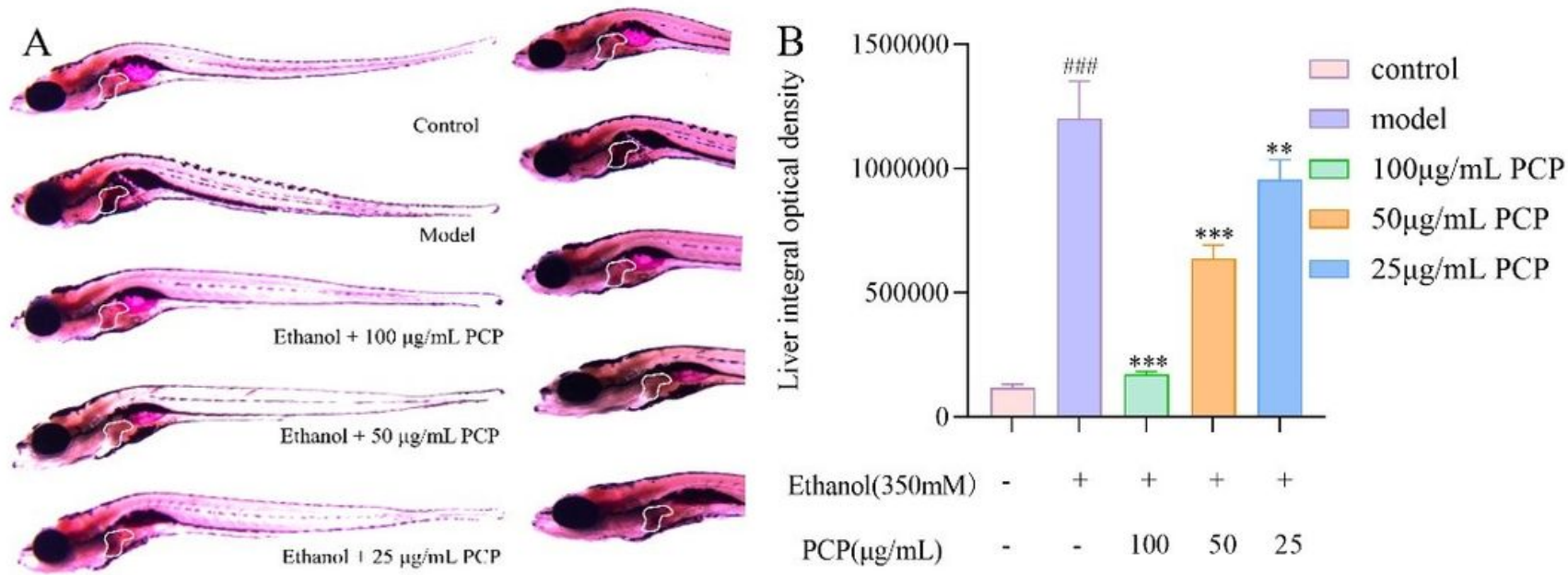

\section{Figure 4}

(A) the whole-mount oil red $O$ staining of larvae zebrafish (the white a indicates the liver). (B) The percent of integrated optical density (IOD) of the area (statistical analysis by Image Pro Plus 6.0 software). Data expressed as the mean \pm SD. \#\#\#P $<0.01$ compared with control group; $* \star * P<0.001, * * P<0.05$ compared with model group

A

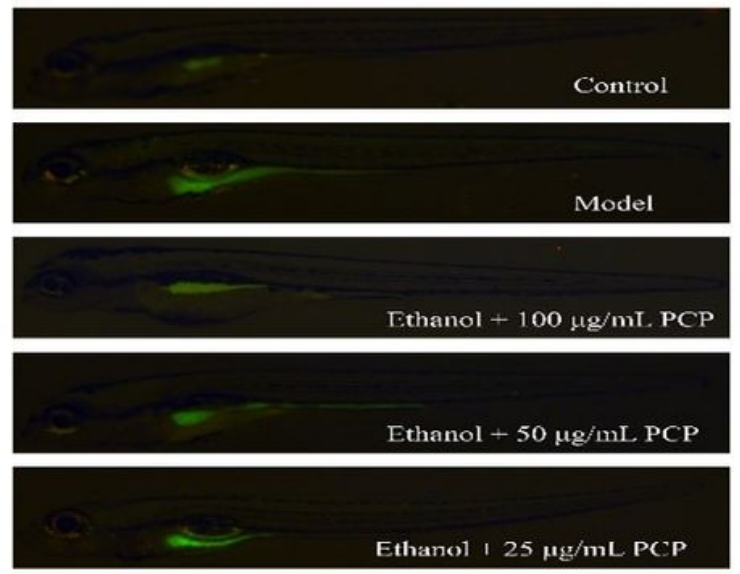

B

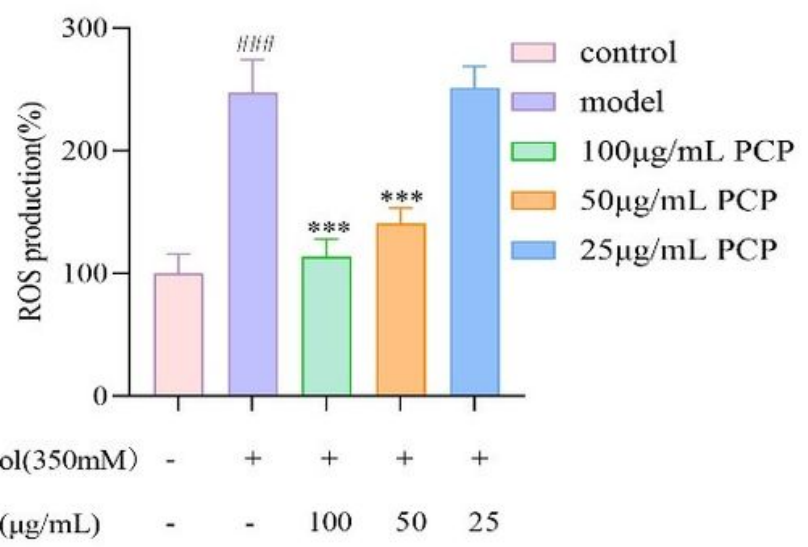




\section{Figure 5}

(A) Fluorescence micrographs of ROS in zebrafish larvae. (B) The distribution and amounts of superoxide anions were quantified according to the fluorescence intensity. Data expressed as the mean $\pm \mathrm{SD}$. \#\#\#P< 0.001 compared with control group; ${ }^{\star \star *} \mathrm{P}<0.001$ compared with model group.

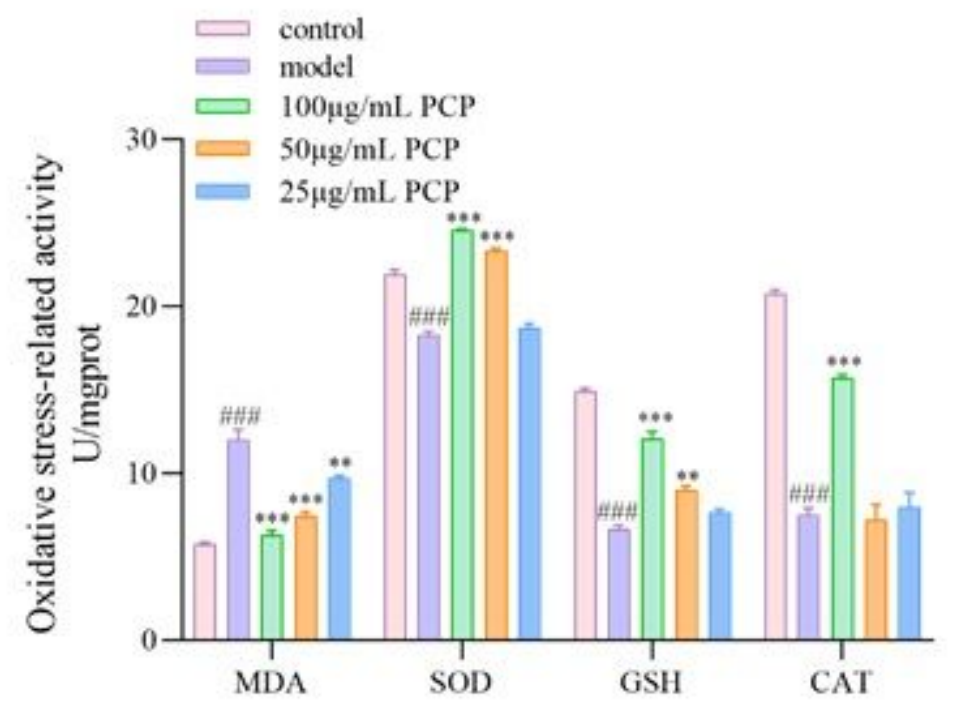

Figure 6

Different doses of PCP effect on serum biochemical indicators of liver function. The following six liver function markers in the serum were assayed: MDA, SOD, GSH, CAT. Data expressed as the mean \pm SD. $\# \# \# P<0.01$ compared with control group; ${ }^{\star \star \star} \mathrm{P}<0.01$, $* * \mathrm{P}<0.05$ compared with model group 

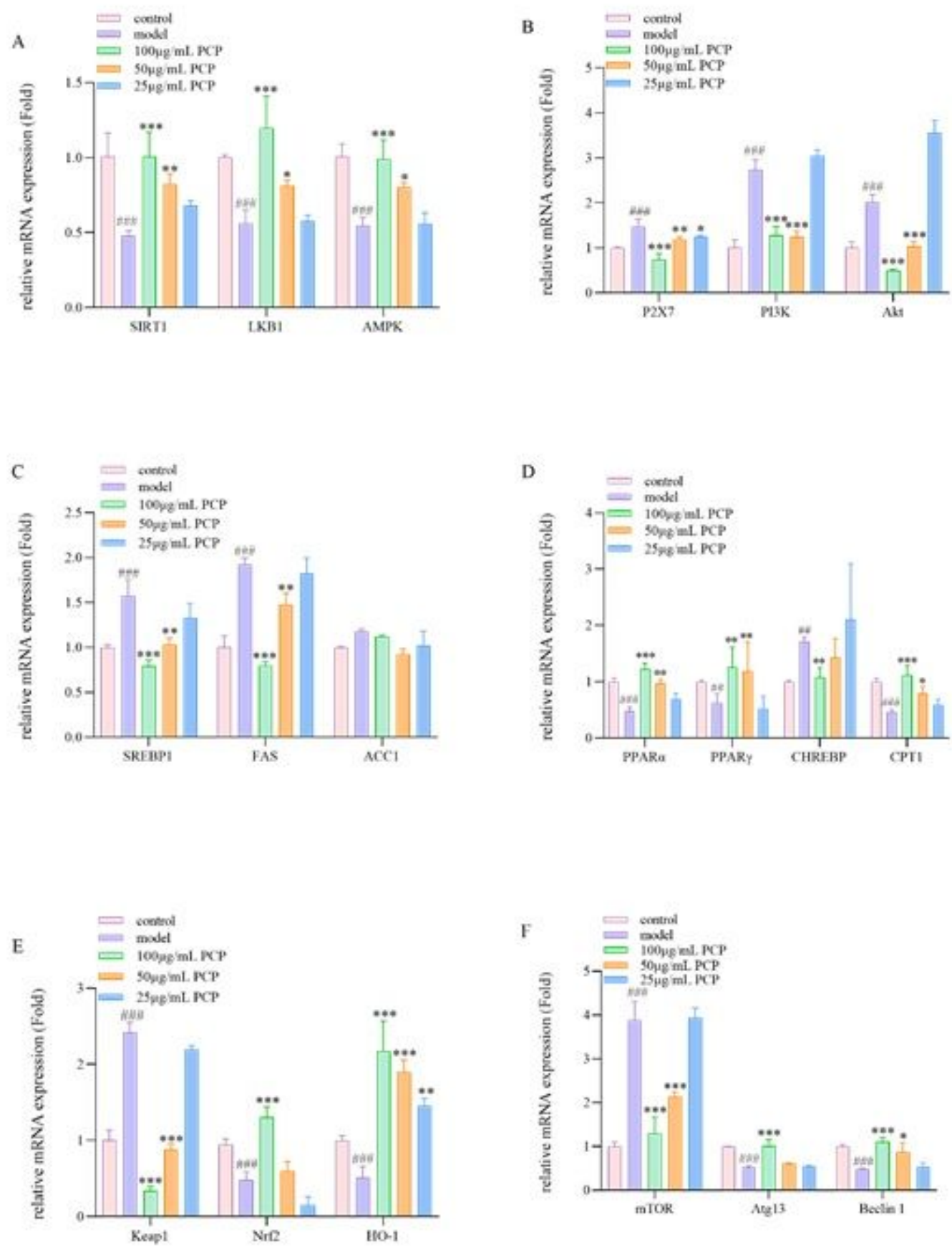

Figure 7

Effect of PCP on genes expression profiles. (A) mRNA expression of SIRT1, LKB1, AMPK on larvae zebrafish; (B) mRNA expression of P2X7, PI3K, Akt on larvae zebrafish; (C) mRNA expression of SREBP1, FAS, ACC1 on larvae zebrafish; (D) mRNA expression of PPARa, PPARy, CHREBP, CPT1 on larvae zebrafish; (E) mRNA expression of Keap1, Nrf2, HO-1 on larvae zebrafish; (F) mRNA expression of mTOR, Atg13, Beclin1 on larvae zebrafish. Data expressed as the mean \pm SD. \#\#\#P $<0.001$ compared with control group; ${ }^{\star \star \star} \mathrm{P}<0.001,{ }^{\star \star} \mathrm{P}<0.01,{ }^{*} \mathrm{P}<0.05$ compared with model group 

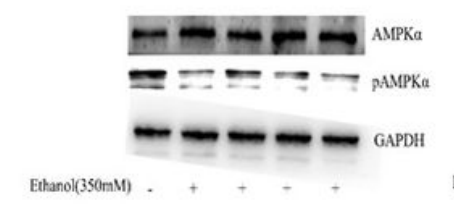

PCP(wyinl)

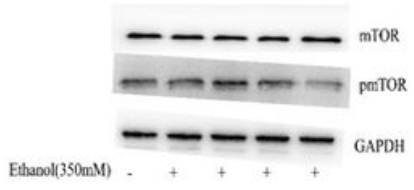

PCP( $\mu g \mathrm{~mL})$

$100 \quad 50 \quad 25$
- - - $\mathrm{ACCl}$

$\longrightarrow-\cdots$ paCCl

$-\infty$ GAPDH

Ethanol(350mM) - + + + +

PCP(ugmL)

$100 \quad 50 \quad 25$

$\because m+\cdots \mathrm{PI}$

$----\infty \mathrm{pPIKK}$

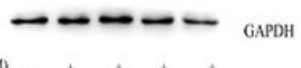

Ethanol(350 $\mathrm{nM})$

PCP(ueglm) - * $100 \quad 50 \quad 25$
A

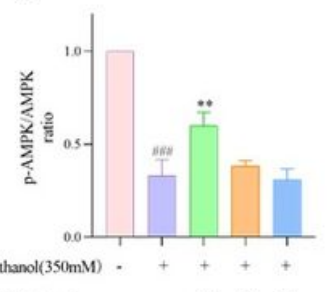

$\begin{array}{lllll}\mathrm{PCP}(\mu \mathrm{g} / \mathrm{mL}) & - & 100 & 50 & 25\end{array}$

C

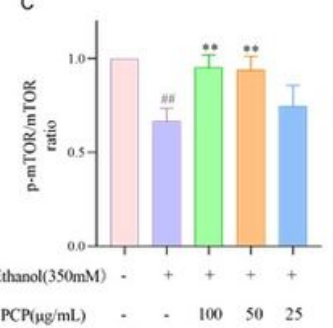

B

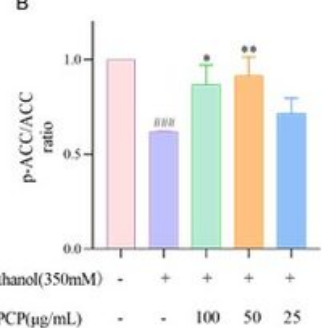

$\begin{array}{lllll}\mathrm{PCP}(\mu \mathrm{g} / \mathrm{mL}) & -\quad & 100 & 50 & 25\end{array}$

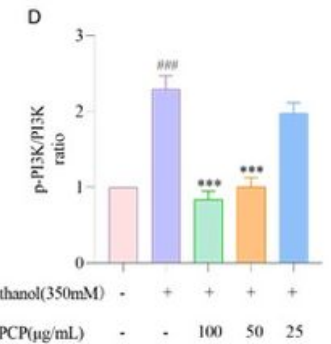

Figure 8

Effect of PCP on proteins expression profiles. Quantitation of western blot analysis of (A) p-AMPK/AMPK, (B) $\mathrm{p}-\mathrm{ACC} / \mathrm{ACC},(\mathrm{C}) \mathrm{p}-\mathrm{mTOR} / \mathrm{mTOR}$, (D) p-PI3K/PI3K. Data expressed as the mean $\pm \mathrm{SD}$. \#\#\#P<0.001 compared with control group; ${ }^{\star \star \star} \mathrm{P}<0.001,{ }^{\star \star} \mathrm{P}<0.01$, ${ }^{\star} \mathrm{P}<0.05$ compared with model group 
A

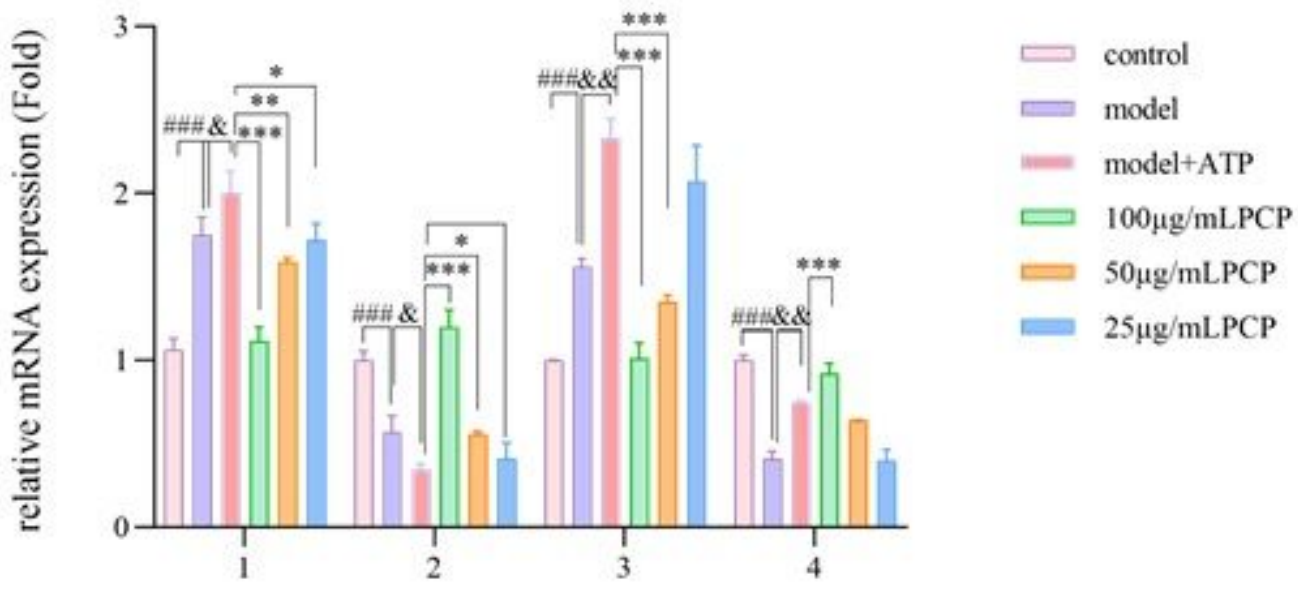

B

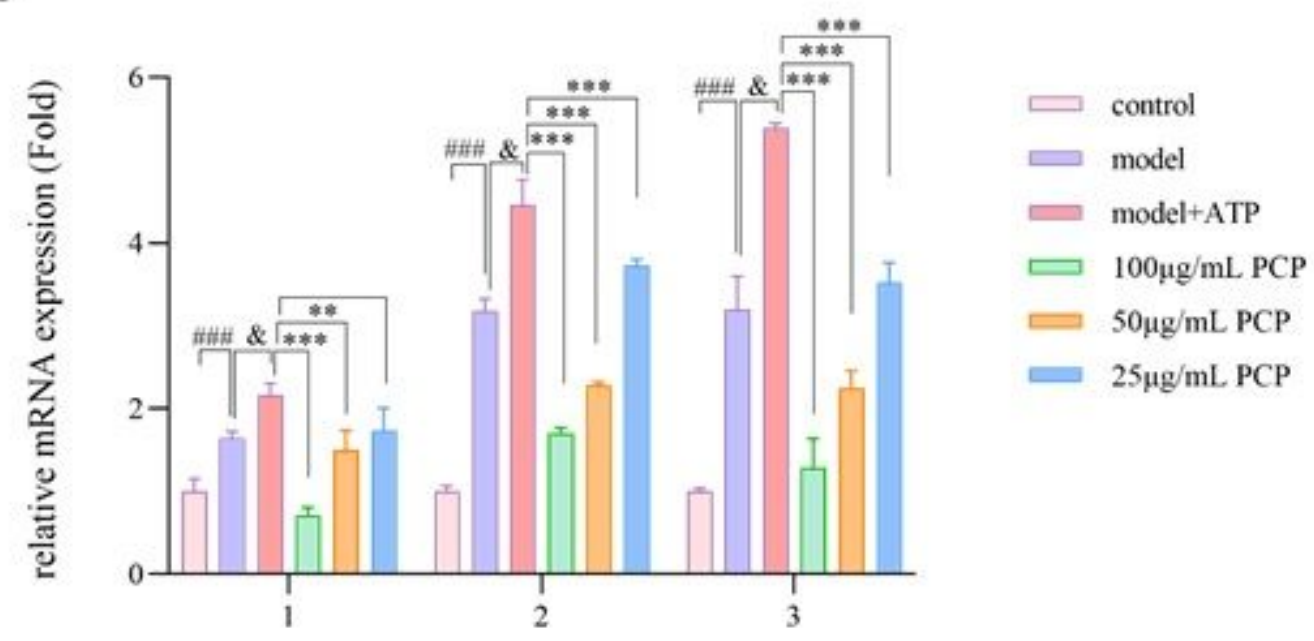

Figure 9

Effect of PCP on mRNA expression profiles. (A) mRNA expression of P2X7, AMPK, Keap1, Nrf2 on larvae zebrafish; (B) mRNA expression of mTOR, PI3K, Akt on larvae zebrafish; Data expressed as the mean \pm SD. \#\#\#P $<0.001$ compared with control group; \&\&P $<0.01$, \&P $<0.05$ compared with model group, $* \star * P<$ $0.001, * * \mathrm{P}<0.01,{ }^{*} \mathrm{P}<0.05$ compared with model+ATP group 


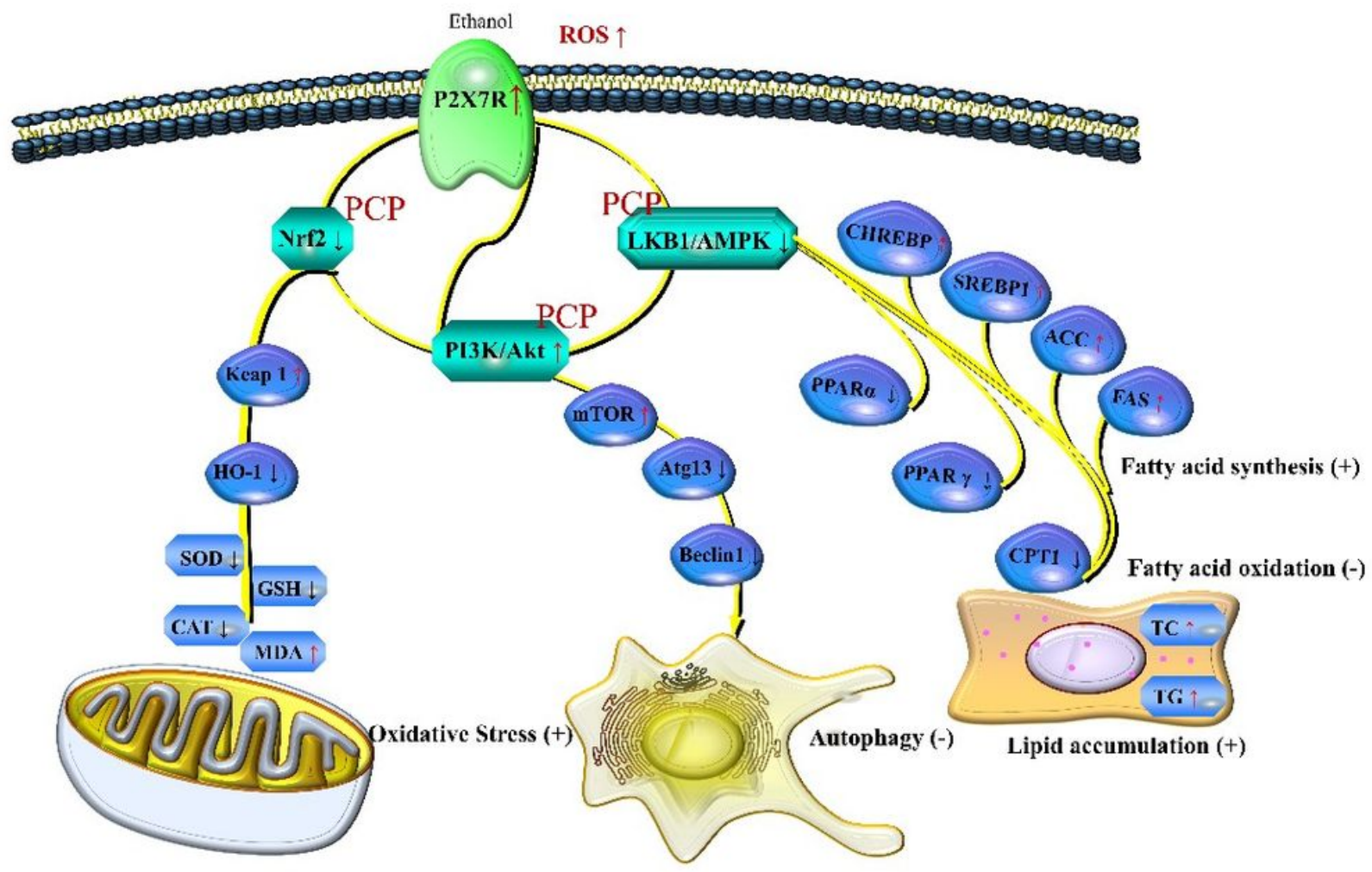

Figure 10

The potential mechanism of PCP on ethanol-induced hepatic steatosis in zebrafish

\section{Supplementary Files}

This is a list of supplementary files associated with this preprint. Click to download.

- GraphicalAbstract.jpg

- DATA.pdf 